\title{
Incorporating Electron Microscopy and Microanalysis into the Engineering Curricula
}

\section{A. Dempere}

Major Analytical Instrumentation Center, University of Florida, Gainesville, FL 32611-6400

With the critical limitations in credit hours, topics, and time imposed upon the undergraduate engineering curricula, it is critical to identify possible routes to get engineering students exposed to the fundamentals and applications of materials characterization and analysis techniques. Thus, this article is intended to describe a specific approach to the introduction of microscopy and microanalysis techniques to one of the courses taken by undergraduate engineering students.

The undergraduate program in the Materials Science and Engineering department at the University of Florida requires its junior students to take the course "Analysis of the Structure of Materials". This course follows a reverse engineering approach which provides an excellent opportunity to get engineering students involved in the use and applications of electron microscopy and microanalysis techniques in the engineering world.

The course requires the students to disassemble an engineered product, characterize and analyze the structure of its components (metals, ceramics, polymers, electronic materials, and composites), and correlate the results of their analysis with material properties and processing techniques used, addressing the criteria used for materials selection by the design engineers of the product.

The course, offered in a lecture and lab format, introduces the students to the history of electron microscopy, the principles and fundamentals of operation and functioning of transmission and scanning electron microscopes, and to microanalysis techniques, including Energy Dispersive Spectroscopy (EDS), Wavelength Dispersive Spectrometry (WDS) and Auger Electron Spectroscopy (AES). Thus, the concepts of resolution, magnification, depth of field, chromatic and spherical aberration, astigmatism, and electron beam parameters, among other, are explained and described in contrast with the optical microscope. As part of the laboratory experience, students learn about digital imaging, how to interpret topographic and compositional images, and get introduced to the concepts of brightness, contrast, and the potential sources of error in conducting measurements on digital images.

The hands-on component of the lab consists in getting the students involved in the disassembling process of a selected engineering device, the selection of samples for analysis from the disassembled components, and sample preparation methods for a variety of materials. In addition, the students get exposed to the principles and operation of the evaporation and sputter coating techniques for the sample preparation of their non-conductive samples.

One of the most critical components of the course is getting the students exposed to the process of data reduction and analysis. Therefore, the students get exposed to the guidelines and best practices for conducting qualitative and quantitative microanalysis, including selection, use and preparation of standards, selection of data correction factors, as well as limitations, advantages and disadvantages of the microanalysis techniques used in the lab. 